\title{
A Trustworthy Framework for Resource-Aware Embedded Programming
}

\author{
Adam D. Barwell \\ adb23@st-andrews.ac.uk \\ University of St Andrews \\ Scotland, UK
}

\author{
Christopher Brown \\ cmb21@st-andrews.ac.uk \\ University of St Andrews \\ Scotland, UK
}

\begin{abstract}
Systems with non-functional requirements, such as Energy, Time and Security (ETS), are of increasing importance due to the proliferation of embedded devices with limited resources such as drones, wireless sensors, and tablet computers. Currently, however, there are little to no programmer supported methodologies or frameworks to allow them to reason about ETS properties in their source code. Drive is one such existing framework supporting the developer by lifting non-functional properties to the source-level through the Contract Specification Language (CSL), allowing non-functional properties to be first-class citizens, and supporting programmerwritten code-level contracts to guarantee the non-functional specifications of the program are met. In this paper, we extend the Drive system by providing rigorous implementations of the underlying proof-engine, modeling the specification of the annotations and assertions from CSL for a representative subset of C, called IMP. We define both an improved abstract interpretation that automatically derives proofs of assertions, and define inference algorithms for the derivation of both abstract interpretations and the context over which the interpretation is indexed. We use the dependentlytyped programming language, Idris, to give a formal definition, and implementation, of our abstract interpretation. Finally, we show our well-formed abstract interpretation over some representative exemplars demonstrating provable assertions of ETS.
\end{abstract}

\section{KEYWORDS}

Dependent Types, Idris, Lightweight Verification, Non-Functional Properties, Abstract Interpretation, Proof-Carrying Code, Embedded Systems

\section{ACM Reference format:}

Adam D. Barwell and Christopher Brown. 2020. A Trustworthy Framework for Resource-Aware Embedded Programming. In Proceedings of International Symposium on Implementation and Application of Functional Languages, Singapore, September 2019 (IFL'19), 12 pages.

https://doi.org/10.1145/1122445.1122456

Permission to make digital or hard copies of all or part of this work for personal or classroom use is granted without fee provided that copies are not made or distributed for profit or commercial advantage and that copies bear this notice and the full citation on the first page. Copyrights for components of this work owned by others than ACM must be honored. Abstracting with credit is permitted. To copy otherwise, or republish, to post on servers or to redistribute to lists, requires prior specific permission and/or a fee. Request permissions from permissions@acm.org.

IFL'19, September 2019, Singapore

(C) 2020 Association for Computing Machinery.

ACM ISBN 978-x-xxxx-xxxx-x/YY/MM...\$15.00

https://doi.org/10.1145/1122445.1122456

\section{INTRODUCTION}

Programs that consider non-functional properties, such as energy consumption or maximum execution time, are of increasing importance due to the proliferation of devices with limited resources; e.g. embedded medical devices, camera pills, drones, wireless sensors, mobile phones and tablets. While conventional understanding of software correctness pertains to the functional properties of a program, such as the absence of errors and bugs, resourcelimited embedded devices prompt additional conformance to nonfunctional requirements [23]. A system that does not conform to its non-functional specification may ultimately render the system useless, or worse, a potential danger to others; e.g. a drone depleting its battery before it can land safely will crash to the ground. It is therefore necessary to develop such systems with an awareness of, and a demonstration of conformity to, their (non-functional) specification.

Drive [6], is a framework for capturing, and reasoning about, non-functional properties such as Energy, Time and Security (ETS) in $\mathrm{C}$ programs. It includes the Contract Specification Language (CSL), an Embedded Domain Specific Language (EDSL) that defines C-statement annotations in order to capture non-functional properties of the statements they annotate, including energy usage, worst-case execution time (WCET), and the degree of vulnerability to side-channel attacks. Drive also facilitates reasoning about non-functional properties via assertion annotations; e.g. whether a statement can be executed within an energy budget (Listing 1). Previously, these contracts were automatically verified using a light-weight abstract interpretation implemented in Idris [5]. This lightweight approach to verification is a form of proof-carrying code [25] since the abstract interpretation automatically derives a proof of whether each assertion holds true for a given context.

In this paper we extend the Drive framework, creating a trustworthy and meaningful proof system for the CSL assertions and ETS properties. Specifically, we provide a rigorous implementation of the abstract interpretation. We model a larger subset of the $\mathrm{C}$ language, IMP, facilitating the inference of necessary contextual information by which our generated proofs are now indexed. We define a big-step operational semantics of assertion annotations in conjunction with the semantics of IMP. We additionally parameterise our abstract interpretation over the type of numeric values. Consequently, proofs are no longer limited to natural numbers, but can be generated, e.g., for integers and real numbers, given a suitable representation. Finally, we demonstrate our executable formalisation on a representative example, capturing a range of programmer-provided assertions and non-functional properties, and demonstrable proofs of these assertions. In line with the CurryHoward correspondence $[28,30]$, we formulate our definitions of 
language, properties, rewrites, and logical and arithmetic formulæ as types, and transformations over types that enact rewrites or determine proofs of properties as total functions. Type-checking ensures the soundness of these functions relative to the definitions given as types, thus ensuring soundness of abstract interpretation and context inference.

\subsection{Contributions}

(1) We present an abstract interpretation of C, extended with CSL assertions, fully implemented in Idris, by deriving and implementing a small general imperative language, called IMP.

(2) We define and implement a big-step operational semantics for well-formed IMP programs and CSL assertions, thereby facilitating robust inference of necessary contextual information in order to generate proofs for CSL assertions.

(3) We present an implementation, in Idris, of an inference system that automatically (dis)proves programmer-provided assertions in IMP, potentially making use of captured nonfunctional information provided by the capture annotations in CSL.

\section{BACKGROUND}

\subsection{The Contract Specification Language}

The Contract Specification Language (CSL) [6] is an embedded domain-specific language developed collaboratively between the University of St Andrews and Inria, Rennes. CSL extends C with special annotations for both capturing non-functional information about source code, and the ability to make assertions (or contracts) using the captured information.

Listing 1 shows an extract from the Levenshtein Distance algorithm, as defined in the BEEBs benchmarks [27] Two CSL capture annotations and an assertion have been added to the code. The capture annotations at Lines 19 and 23 direct the compiler to invoke a worst-case execution time (WCET) analysis for the assignment statements on Lines 20 and 24-26, respectively. The results of these analyses are assigned to the declared variables that are passed to the capture annotations; i.e. true_time and false_time. These measurements could be used by the programmer as a simple or coarse-grained check for vulnerability to side-channel attacks; i.e. using differences in execution time to infer information about secret data [20]. The assertion at Line 31 expresses this check: the implementation is vulnerable if the assertion does not hold true.

Brown et al. define assertion expressions as being standard Boolean expression evaluating to true or false. The proof of whether an assertion holds true within a given context (i.e. mapping of variables to values) is inferred by a simple decision procedure implemented in Idris.

\subsection{Dependent Types}

Dependently typed languages take advantage of the Curry-Howard correspondence, which states that, given a suitably rich type system, (certain kinds of) proofs can be represented as programs [30]. For languages with insufficiently rich type systems, such as $\mathrm{C}$, dependently-typed languages can be used to produce an abstract interpretation [11] of a given program in those languages. Such
Listing 1: An extract of the Levenshtein algorithm from the BEEBs benchmarks suite for $\mathrm{C}$

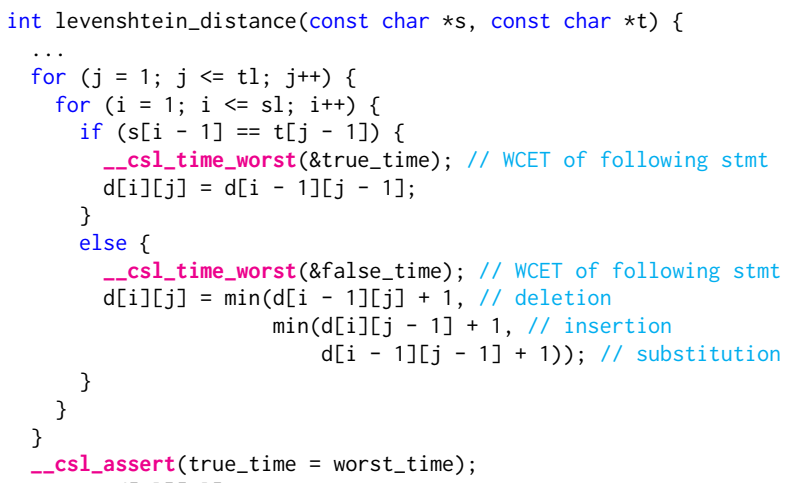

abstract interpretations can be used to derive proofs of desired properties [2].

In the case of dependently-typed languages, under the propositions as types view, dependent types are used to represent predicates [31]. For example, (Even : ( $n:$ Nat) $\rightarrow$ Type) defines the type of evidence (or proofs) that a natural number, $n$, is even. In cases where the property does not hold true, e.g. Even 1, and assuming a suitably restricted definition of that property, the type is uninhabited. An uninhabited type represents falsity. Evidence that a predicate does not hold true can be represented by the type function, (Not $a=a \rightarrow$ Void), where $a$ is a type variable and void is the empty type; i.e. it has no constructors. Using dependent types in this way, properties that represent a (non-)functional specification can be encoded as predicates (i.e. types). Accordingly, total functions, $f: A \rightarrow B$, allow for the derivation of evidence that the predicate $B$ can be constructed given evidence of A. Type-checking ensures the soundness of these functions [28].

We take advantage of the above features by implementing our system in the dependently-typed language Idris, a functional language developed at the University of St Andrews [5]. The syntax of Idris is similar to Haskell [19], and like Haskell, Idris supports algebraic data types with pattern matching, type classes, and donotation. Unlike Haskell, Idris evaluates its terms eagerly. Definitions, e.g. of languages and well-formedness, are defined by giving their definitions as types in Idris. For example, the aforementioned Even predicate can be defined:

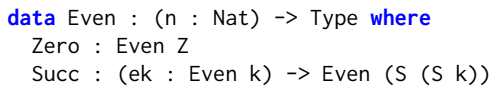

where, Even is the name of the type being defined, Nat is the type of natural numbers, ( $n$ : Nat) is a (named) argument to the type, and both zero and succ are constructors. Constructors may have (named) arguments; e.g. (ek : Even k). Constructors may also restrict the values of their arguments; e.g. Zero explicitly states that $n=0$, and Succ states that $n=S(S k)(i . e . k+2)$, given an inhabitant of Even $k$. As is desired, under this definition, there is no way to construct an element of, e.g., (Even 1) using either constructor. 
In order to determine whether (Elem $n$ ) is inhabited for a given $n$, we define the function isEven.

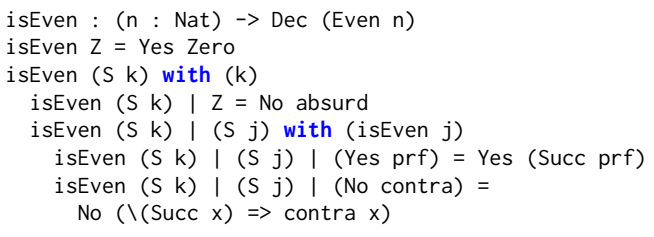

Here, Dec is the type for a decidable property, where yes holds a proof of the property and No holds a proof of its contradiction. The with rule is used to pattern match on intermediate values, similar to a case expression in Haskell. The function (absurd : Uninhabited $t \Rightarrow t \rightarrow$ a) is a convenience function for contradictions where a type, here (Even 1), is uninhabited. The argument to No on Line 8 is a function of type (Even ( $(S j)$ ) $\rightarrow$ Void) and represents a contradiction of (Even $(S(S j)$ )) when (Even $j$ ) is uninhabited. Since this is a total definition, as guaranteed by the type-checker, isEven is a decision procedure for the type (predicate) (Elem $n$ ) for all values of $n$.

\subsection{Non-Functional Properties}

The Drive system focuses on three non-functional properties that we consider to be the most commonplace. The first two are time and energy, and the third property, security, which we do not consider in this paper, is typically one that is required as time and energy properties are leaked from a program and used by adversaries to obtain information about the algorithm. In Drive, measurements for these non-functional properties are provided by third-party tools and models. In this paper, we omit the details of obtaining such non-functional information, leaving it for future work to extend the Drive system with support to link to automated tools to obtain the information automatically.

For time, we consider the worst-case execution time (WCET) obtained by executing the code with various underlying profiling tools such as the WCC compiler produced by the University of Hamburg [12].

Energy measurements are typically obtained by using a model such as those provided by Eder et al. [24] or by measuring the amount of energy in Joules $(\mathrm{J})$ that is used by a complete processor package; i.e. by measuring the total energy that is drawn by each hardware CPU socket, and energy usage is typically calculated by computing the rate of change in power per unit of time using the formulae shown below:

$$
\begin{aligned}
\text { Energy } & =\text { Power } \times \text { Time } \\
\text { foules } & =\text { Watts } \times \text { Seconds }
\end{aligned}
$$

\section{REPRESENTING WELL-FORMED PROGRAMS IN IDRIS}

In this section we introduce and define a simple imperative language, IMP, in order to facilitate the definition of our semantics for CSL annotations and assertions. Accordingly, we define an

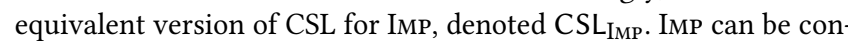
sidered a modified standard While language [26], adding arrays and restricting iteration. In principle, IMP can be extended with

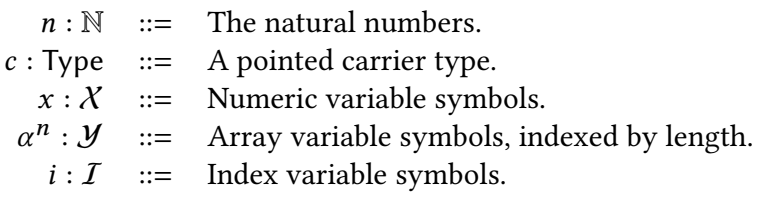

Figure 1: Key to terms and notation.

additional language constructs, such as tuples in order to allow the representation of a wider range of $C$ programs in IMP. In the following sections, Idris code snippets have been simplified using mathematical notation where possible to aid clarity ${ }^{1}$.

\subsection{Variable Representation}

Variables are represented as three disjoint sets: $\mathcal{X}$ denotes the set of numeric variable symbols, $\mathcal{Y}$ denotes the set of array variable symbols; and $\mathcal{I}$ denotes the set of index variable symbols for accessing elements in arrays. This separation trivially ensures that array variables are not used where an arithmetic expression is expected, and vice versa. Index variable symbols are used to facilitate checking that array accesses are not out of bounds.

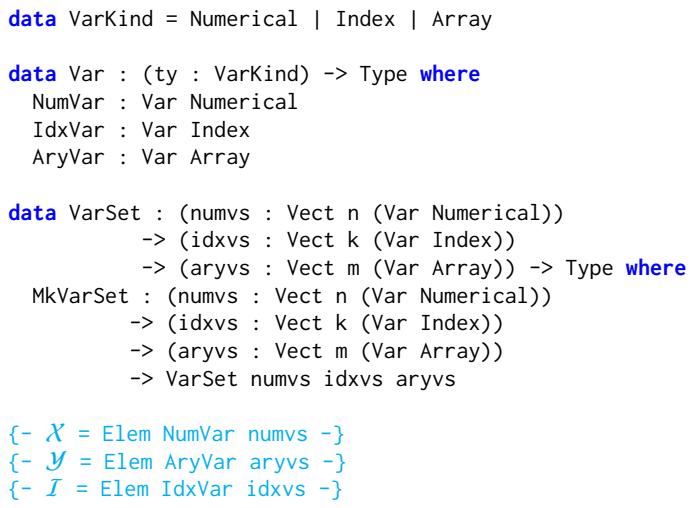

In our Idris implementation, variables are represented using proofs of existence in a vector; e.g. $X=$ Elem NumVar numvs. Here, numvs is a vector of $\mathrm{n}$ elements with type var Numerical, where Numeric defines the set to which the variable belongs. Variables in $\mathcal{I}$ and $\mathcal{Y}$ are defined analogously. Each array variable, $\alpha^{n}: \mathcal{Y}$, is indexed by the length of the array that it represents, $n: \mathbb{N}$, where $n>0$. Array sizes occur at the expression and statement level in our implementation. Index variables are used to access elements in arrays; they are mapped to natural numbers and may only be incremented. An array access expression, e.g. $\alpha^{n}[i]$, is deemed to be out-of-bounds when $i \geq n$.

Data definitions representing syntax are all indexed by the type Varset, which is functionally equivalent to the triple $(\mathcal{X}, \mathcal{I}, \mathcal{Y})$, and allows variables to be used in IMP programs. In order to simplify our presentation, we use $x, x_{1}, x_{2}, \cdots: \mathcal{X}, i, i_{1}, i_{2}, \cdots: \mathcal{I}$, and $\alpha^{n}, \alpha_{1}^{m}, \alpha_{2}^{k}, \cdots: \mathcal{y}$ to represent variables.

\footnotetext{
${ }^{1}$ Our full implementation can be found at https://github.com/adbarwell/IFL2019-Drive
} 
Example 3.1 (Numeric Variables). The numeric variables, $x_{1}, x_{2}$ : $\mathcal{X}$, can be represented in our implementation via the definitions: $x_{1}=$ Here and $x_{2}=$ There Here, where numvs $=[$ NumVar, NumVar,$\ldots]$.

\subsection{Numeric Values}

Instead of defining IMP with a specific numeric data type, e.g. the natural numbers, as in [6], we aim to build a framework that allows for generic representations of numeric values. Taking inspiration from Slama and Brady [30], we index expressions and statements with a setoid, (set : Setoid c $(\simeq)$ ) and an algebraic structure, (Struct c set kind), defined on a carrier type, (c : Type). Our implementation differs from Slama and Brady's in that we define both setoid and algebraic structures as data types instead of interfaces in order to simplify their use as constraints in other data type declarations. Additionally, we extend our setoid definition with both a zeroth element, zero, that is used as a default value for array elements, and a boolean equivalence operator that is required to be equivalent to $(\simeq)$; i.e. $x_{1} \equiv x_{2}$ iff $x_{1} \simeq x_{2}$ for all $x_{1}, x_{2}: c$. Due to this requirement, we do not also require proofs of symmetry, transitivity, etc. for $\equiv$.

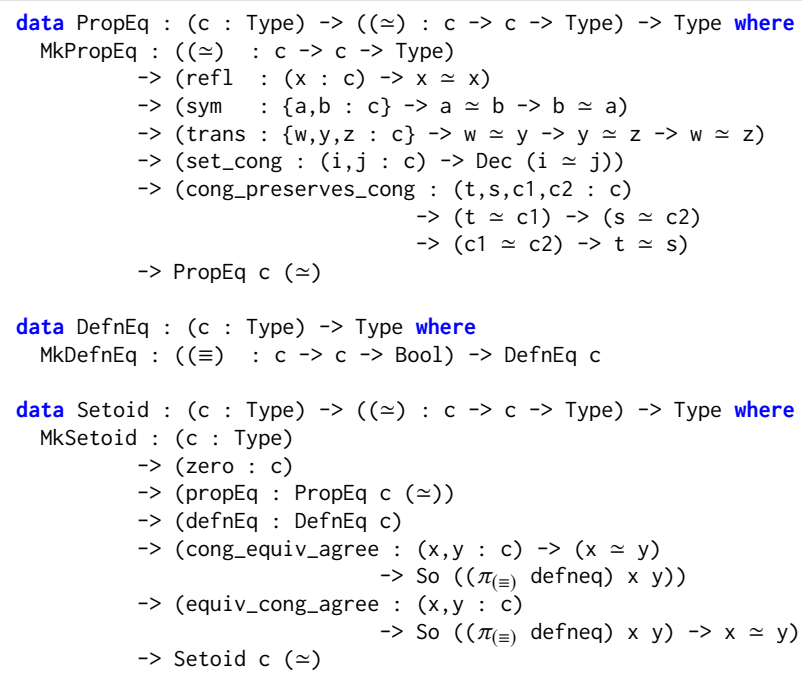

We define IMP for six fundamental algebraic structures, Magma, Semigroup, Monoid, Group, AlebelianGroup and Ring, shown in the following listings. Algebraic structures are defined in the type struct.

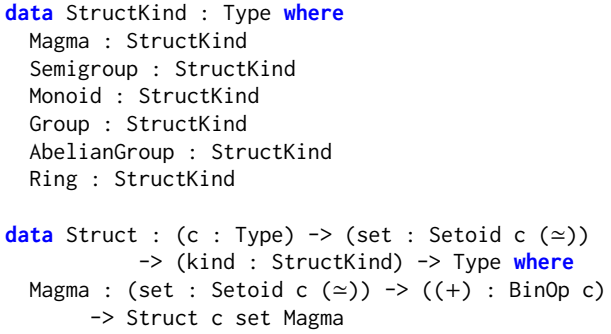

We define an ordering over these algebraic structures, where Magma $<$ Semigroup < Monoid < Group < AbelianGroup < Ring. This ordering is used to ensure that we can only attempt to project functions that express a structure's requirements from structures that define them; e.g. the (additive) identity element from monoids or greater. Our implementation can be extended with additional structures, e.g. fields, or a total ordering over $\mathrm{c}$ that enables inequalities in boolean expressions.

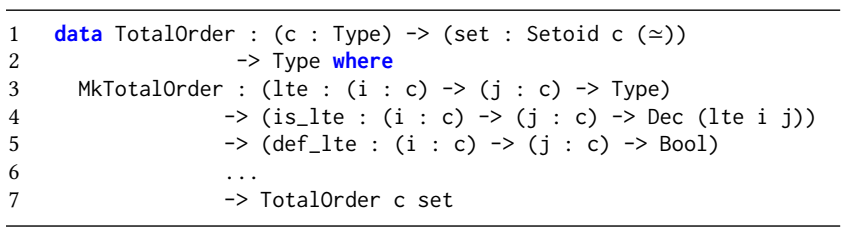

Magma takes a proof that there is a setoid on $\mathrm{c}$ and are equipped with a binary operation, $(+): c \rightarrow c->$.

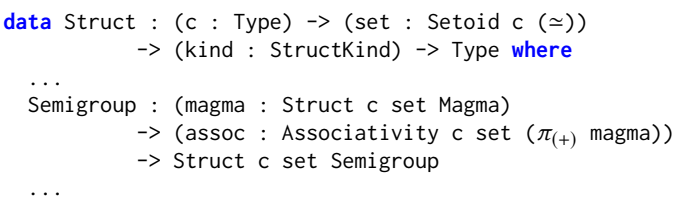

Semigroup (as shown in the listing above at Line 4) extends Magma with the requirement that $(+)$ is associative. We define these requirements via separate data types; e.g. for associativity,

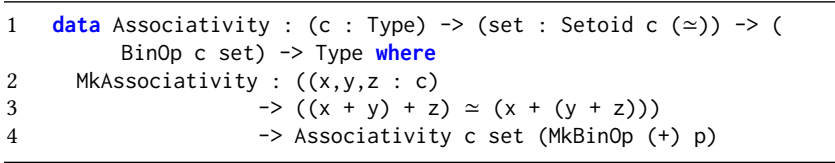

Such property types, have single constructors whose arguments express the relevant requirements. Here, associativity requires a function that calculates an explicit witness of type $((x+y)+z)$ $\simeq(x+(y+z))$, for the given binary operation $(+)$, definition of (propositional) equality $(\simeq)$, and all natural numbers, $x, y$, and $z$. We define $\pi_{(+)}$: Struct $\mathrm{c}$ set $\mathrm{k} \rightarrow$ Binop $\mathrm{c}$ set, used in the definition of Semigroup, inter alia, as a function that projects the binary operation from a Magma.

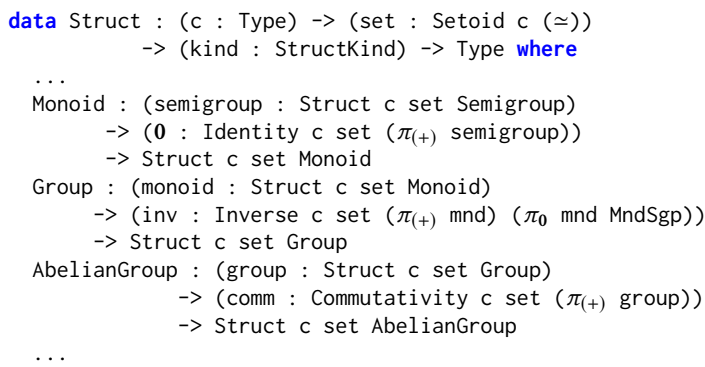

Similar to Magma and Semigroup definitions, a Monoid extends a given Semigroup with the requirement that $c$ has an (additive) identity element, a Group extends a given Monoid with the requirement that there is a function $(-): c \rightarrow c$ that produces the (additive) inverse of its argument, and an AbelianGroup extends a given Group with the requirement that the $(+)$ operation is commutative.

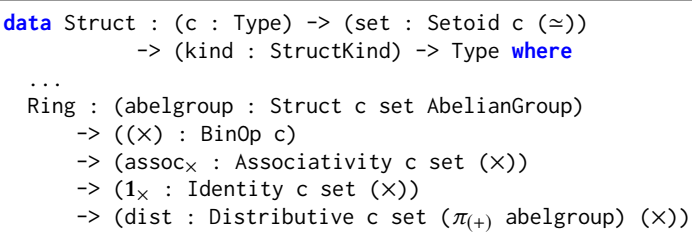


$9 \quad \rightarrow$ Struct $c$ set Ring

In order to illustrate that we are not limited to a single binary operation, we include Ring. A Ring extends a given AbelianGroup with a secondary binary operation over $\mathrm{c}$, denoted $(\times)$, which is associative, has an identity element $\left(\mathbf{1}_{\times}\right)$, and distributes over $(+)$.

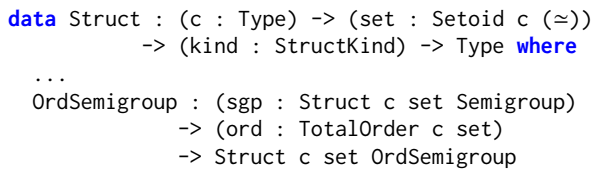

Similarly, we might extend any of the above structures with the total ordering over c. Here, we have extended semigroup.

Example 3.2 (The Natural Numbers as a Semigroup). We can take advantage of the Idris Prelude definitions of propositional equality, addition, and lemmas over the natural numbers in order to define the semigroup structure for natural numbers.

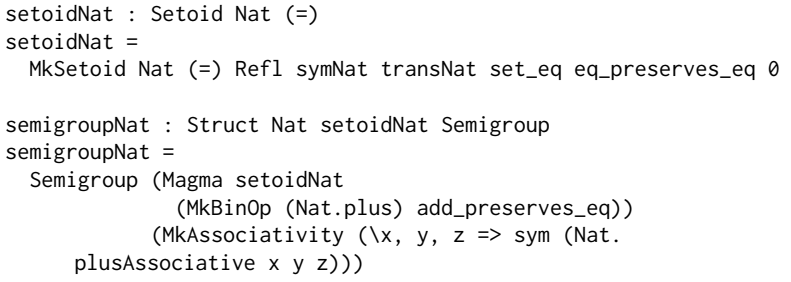

Example 3.3 (The Natural Numbers as an Ordered Semigroup). We can further extend the definition in Example 3.2 with the Idris Prelude definitions of inequalities (LTE).

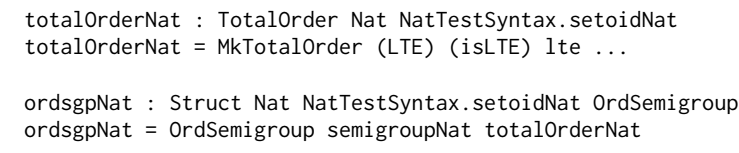

\subsection{Intrinsically Typed Syntax}

Since we are only concerned with well-formed input, it makes sense to restrict the programs that are expressible in IMP as early as possible. Thus, we begin with syntax that is already type-safe; other aspects of well-formedness will be covered in Section 3.4.

3.3.1 Arithmetic Expressions. Arithmetic expressions comprise literal values, numeric variables, array accesses, and an addition operator for all fundamental algebraic structures that we consider. For Group and above, negation is available, representing inverses. Similarly, for Ring, a multiplication operator is available. Arithmetic expressions are defined in our formalism by the type AExp.

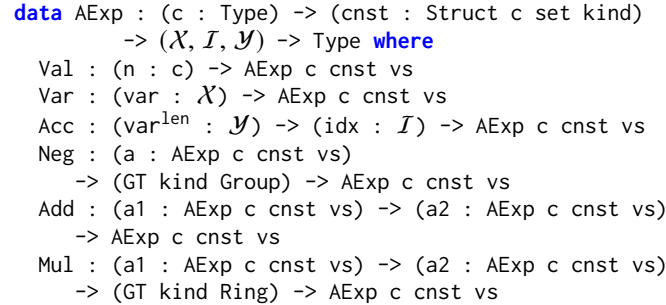

Here, (SKOrd : StructKind $\rightarrow$ StructKind $\rightarrow$ Type) is used to restrict the use of constructors Neg and Mul to when the appropriate algebraic structure is defined over c. We omit this restriction on Add, since the addition operator is defined as a requirement of Magma, the least element in our ordering. Array access expressions, Acc, require the array variable, var, the length of the array being accessed, len, and an index variable, idx. In our presentation, (var: $y$ ) $\rightarrow$ (len: Nat), (varlen : $y$ ), and $\alpha^{n}: \mathcal{Y}$ are all equivalent.

Example 3.4 (Arithmetic Expressions for Natural Numbers). Given the definitions for natural numbers as a Semigroup in Example 3.2, we can define the exemplar arithmetic expression

1 a : AExp Nat semigroupNat $(\mathcal{X}, \mathcal{I}, \mathcal{Y})$

$2 \quad a=\operatorname{Add}(\operatorname{Val} 42)\left(\operatorname{Acc} \alpha_{2}^{5} i_{1}\right)$

Here, we add a literal value to the element in the array $\alpha_{2}^{5}$ at index $i_{1}$. Since a Semigroup is defined over Nat, any occurrences of Neg or Mul in an arithmetic expression will lead to a type error; therefore, such arithmetic expressions cannot be constructed. Any occurrences of a numeric variable or literal in an array access expression, e.g. (Acc $x_{1}$ (Val 42)), or an array variable outside of an array access, e.g. (Add $\alpha_{2}^{5}$ (Var 42)), are similarly invalid.

3.3.2 Boolean Expressions. Boolean expressions comprise equality and inequality comparisons.

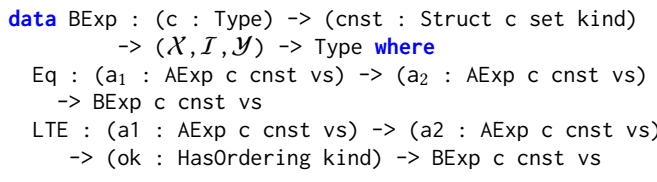

Definitions for both equality and inequalities for a given $c$ are provided by cnst. As with Neg and Mul above, LTE has an additional argument that requires demonstration that a total ordering is defined for the given algebraic structure.

Example 3.5 (Boolean Expressions for Natural Numbers). Given the definitions for natural numbers as a Semigroup in Example 3.2 and an ordered Semigroup in Example 3.3, we can define the exemplar Boolean expressions

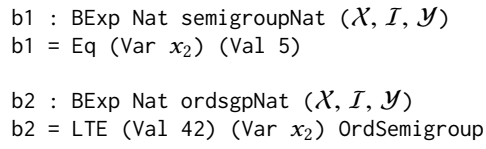

Here, we express $x_{2} \simeq 5$ in b1 and the inequality $42 \leq x_{2}$ in b2. The latter requires the proof, OrdSemigroup, that ordsgpNat is equipped with a total ordering.

3.3.3 Statements. Statements comprise numeric variable assignment, index variable assignment and increment, array declaration and update, for-loops, statement composition, and CSL assertions.

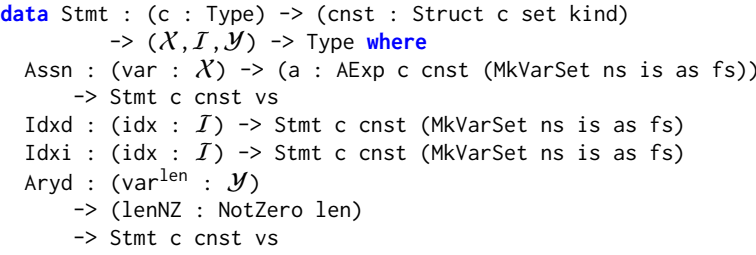




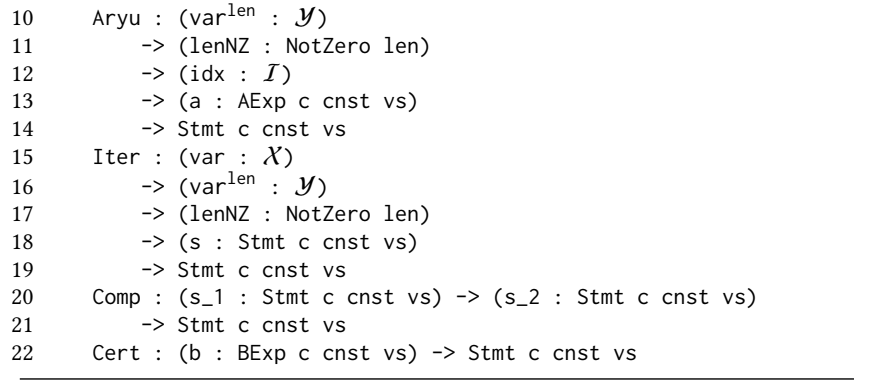

Unlike in arithmetic and Boolean expressions, all statements can be used with all algebraic structures. We assume that any capture annotations have been reified to numeric assignment expressions with literal values prior to the representation in IMP. This leaves assertions, denoted cert in order to avoid confusion with numeric assignment statements, as the sole CSL construct that extends IMP. To simplify our presentation, we use Boolean expressions (Section 3.3.2) as our assertion language. In principle, our the assertion language could be different to Boolean expressions.

Example 3.6 (Statements for Natural Numbers). Given the definitions for natural numbers as a Semigroup in Example 3.2, we can define an exemplar statement.

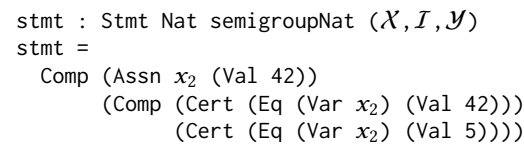

Here, stmt is the composition of three statements: the assignment of $x_{2}$ to 42 , the assertion that $x_{2}$ is equal to 42 , and the assertion that $x_{2}$ is equal to 5 .

\subsection{Well-Formedness}

Since our syntax definitions in Section 3.3 ensure that programs in IMP are type-safe, our well-formedness property is principally concerned with avoiding the occurrence of undeclared variables and out-of-bounds array accesses. Determining well-formedness of arbitrary programs is a two-stage process for expressions and a four-stage process for statements. As a precursor to checking for out-of-bounds array accesses and occurrences of numeric and array variables that have not been declared, we first determine the validity of index variable occurrences and reify them according to a context. Reification produces an equivalent representation of the program with no index variable symbols. For statements, prior to reification, we first convert the statements into a continuation passing style and unroll for-loops. We are able to unroll all possible loops, since loops are defined to iterate over a (finite) fixed-length array.

3.4.1 Environment. An environment, Env, represents the variable symbols that are in scope at a given point in a program.

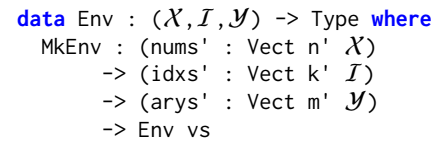

In order to simplify our presentation, we use $\Gamma$ to represent Env. While the terms environment and context are usually used interchangeably, in this paper, we will use environment to refer to the (sub)set(s) of variable symbols that are in scope, and context to refer to functions that map variable symbols to (ground) values.

Example 3.7 (Environment). We define an exemplar environment,

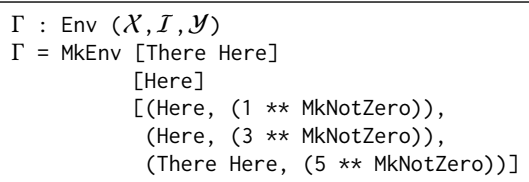

which states that one numeric variable, one index variable, and three array variables have been declared, given the sets of variable symbols $(\mathcal{X}, \mathcal{I}, \mathcal{Y})$. Array variable symbols are, in effect, a pair comprising an element of type Elem Aryvar as and the length of the array. We consider two array variables to be distinct even when the element of type Elem AryVar as is the same but their defined lengths differ. Thus, in the above example, (Here, ( $1 * *$ MkNotZero)) and (Here, $(3 * *$ MkNotZero)) are considered different variables. For convenience and clarity of presentation, we equivalently denote $\Gamma$ as the triple:

$$
\Gamma=\left(\left\{x_{2}\right\},\left\{i_{1}\right\},\left\{\alpha_{1}^{1}, \alpha_{1}^{3}, \alpha_{2}^{5}\right\}\right)
$$

3.4.2 Index Context. Index variables occur only in array access expressions and are incremented in statements. We define an index context, $\Upsilon$, to be a function from index variable symbols to natural numbers. In our implementation, we represent this using the data type Idxctx.

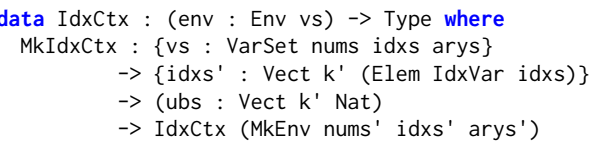

Here, ubs is a vector of natural numbers. When an index variable occurs in the body of a loop, this represents the maximum value it is assigned to. We consider that the $j^{\text {th }}$ element of idxs' is mapped to the $j^{\text {th }}$ element in ubs. In order to determine the $j^{\text {th }}$ element in ubs, we define the function arrIdxBounds that transforms a proof that some index variable is an element in idxs' into a proof that ub is the corresponding element in ubs.

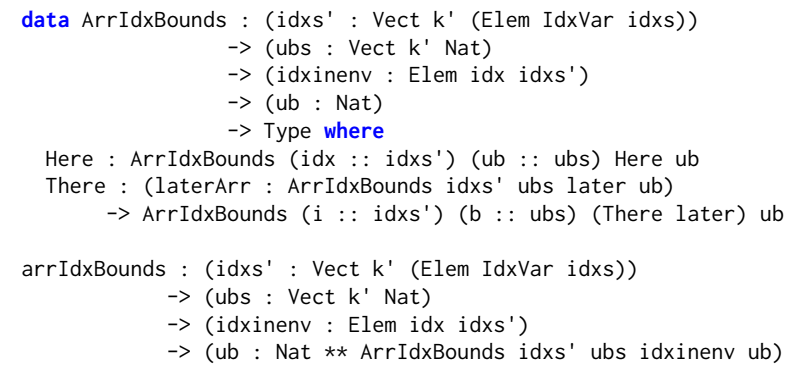

In order to simplify our presentation, we use $\Upsilon$ to represent Idxctx, where $\Upsilon(i)=k$ states that the index variable $i$ is mapped to some $k$ in the context $\Upsilon$. 
Example 3.8 (Index Context). We define an exemplar index context,

$1 \Upsilon: \operatorname{IdxCtx} \Gamma$

$2 \Upsilon=\operatorname{MkIdxCtx}[4]$

assuming the environment $\Gamma$ from Example 3.7, which states that there is a single declared index variable, $i_{1}$. For convenience and clarity of presentation, we equivalently denote $\Upsilon$ using substitution notation:

$$
\Upsilon=\left\{i_{1} \mapsto 4\right\}
$$

\subsubsection{Arithmetic Expressions.}

Index Reification. Since index reification pertains only to array access expressions, the definition of index-reified algebraic expressions only differs from AExp in the Acc case.

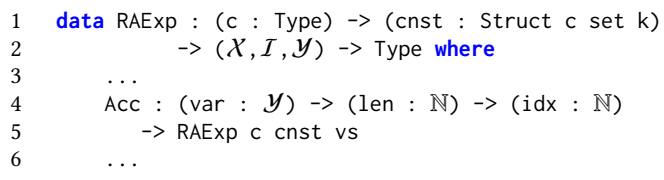

Additionally, we define ReifyIdx to relate an algebraic expression with its reified form. Again, Acc is the only interesting case, where $i d x i s d e c l d$ is a proof that $i d x$ is in scope, and arridxub relates idxisdecld with its upper bound ub.

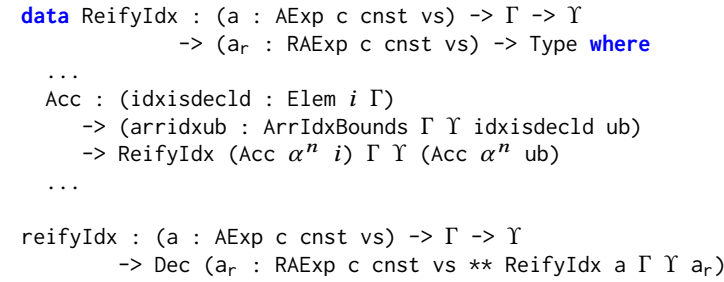

We define reifyIdx to transform an algebraic expression, $a$ into an equivalent reified algebraic expression, $\mathrm{a}_{r}$, given some environment and index context. This is a decision procedure since $i d x$ may not be in scope.

Example 3.9 (Index Reification of Arithmetic Expressions for $\mathbb{N}$ ). Recall the simple arithmetic expression from Example 3.4,

$\begin{array}{ll}1 & \text { a : AExp Nat semigroupNat }(\boldsymbol{X}, \boldsymbol{Y}) \\ 2 & \mathrm{a}=\operatorname{Add}(\operatorname{Val} 42)\left(\operatorname{Acc} \alpha_{2}^{5} \boldsymbol{i}_{1}\right)\end{array}$

The index $i_{1}$ in a can be reified given an Index Context, $\Upsilon$. Assuming $\Upsilon$ as defined in Example 3.8, the index reification, $a_{r}$, of a is:

$1 a_{r}$ : RAExp Nat semigroupNat $(\mathcal{X}, \mathcal{I}, \mathcal{Y})$

$2 \quad a_{r}=$ Add $(\operatorname{Val} 42)\left(\right.$ Acc $\left.\alpha_{2}^{5} 4\right)$

Well-Formedness of Reified Arithmetic Expressions. For arithmetic expressions, var and Acc are the interesting cases.

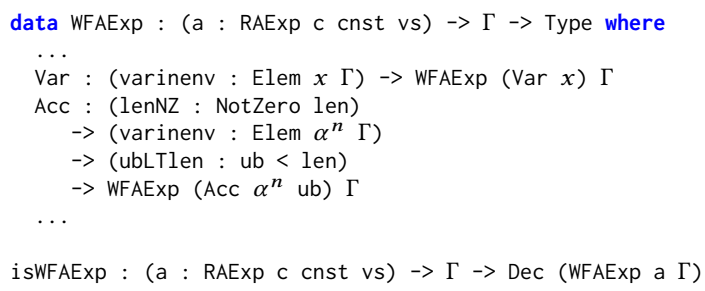

Here, var requires a proof that $x$ is in scope, and Acc similarly requires a proof that $\alpha^{n}$ is in scope, but also that $n>0$ and that the index is not out-of-bounds (i.e. ub $<$ len). Constructs that have subexpressions (i.e. Neg, Add, and Mul) are well-formed only when their subexpressions are well-formed. Literals ( $\mathrm{Val}$ ) are trivially well-formed. The definition of the decision procedure for wellformedness, isWFAExp, is unsurprising.

Example 3.10 (Well-Formedness of Arithmetic Expressions for $\mathbb{N}$ ). We can produce a proof that the reified algebraic expression $a_{r}$ in Example 3.9 is well-formed under the environment defined in Example 3.7.

3.4.4 Boolean Expressions. Both the index-reification and wellformedness of Boolean expressions are trivial. For both equality and inequality expressions, (arithmetic) sub-expressions are themselves reified and checked for well-formedness.

\subsubsection{Statements.}

Continuation Passing Style. In order to facilitate the inference of environments and contexts, statements are first transformed into an equivalent continuation-passing style.

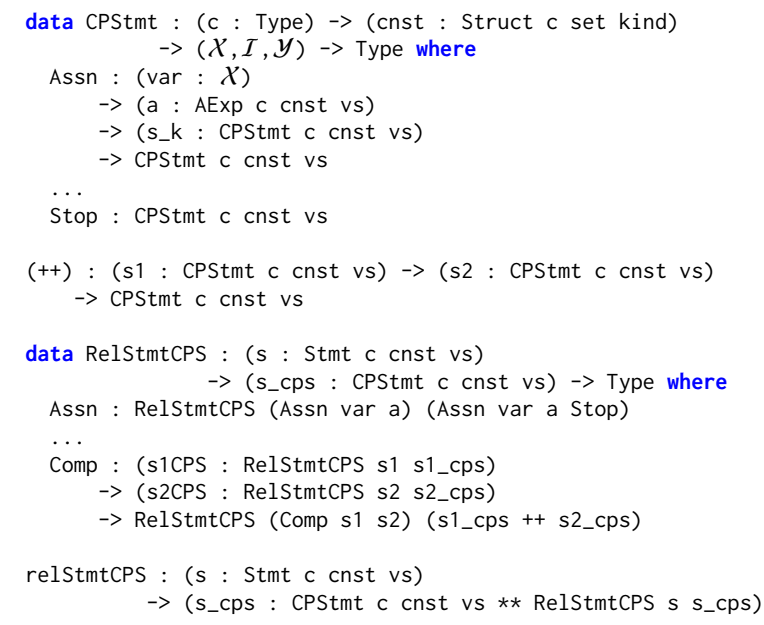

Here, we define a new statement representation, cPStmt, and the type, RelstmtCPS that defines the relation between statements and CPS statements. Statement composition is the only interesting case: s2 is appended to s1, where we define (++) to substitute the instance of stop in s1 for s2 (occurrences of stop in the body of for-statements are not substituted).

Loop Unrolling. Loops in CPS statements are then unrolled. We define a new statement representation, Lustmt that is the same as CPStmt but without loops.

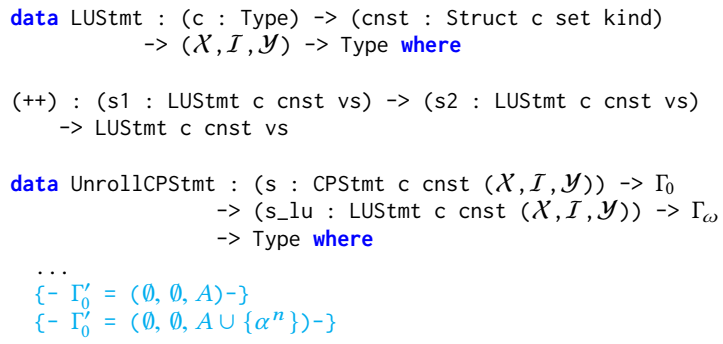




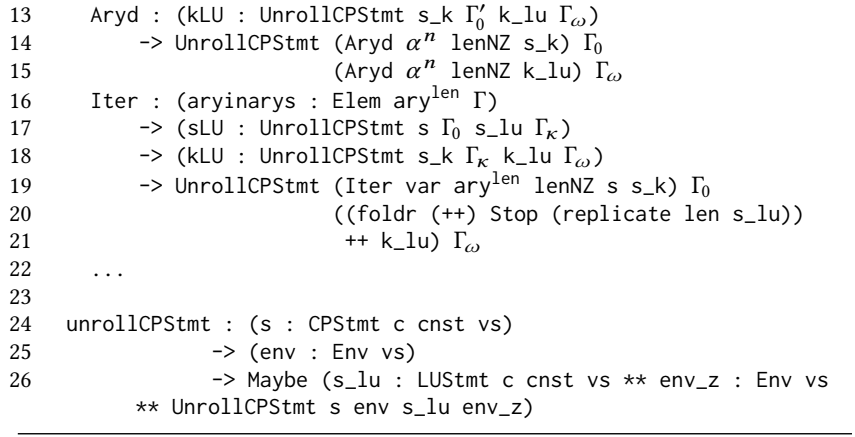

Similar to before, we additionally define a type that relates a CPS statement to its loop-unrolled equivalent. An environment is maintained between statements in order to know the length of the list being iterated over in the for-statement. Since we are only concerned with arrays, only array declarations update the environment. The other interesting case is Iter, which replaces the loop statement, e.g.

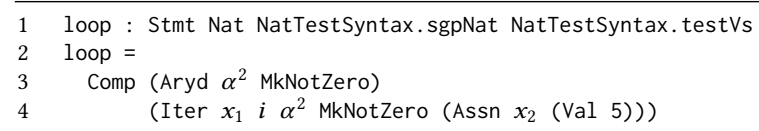

with a declaration of the index variable, $n-1$ repetitions of the loop body prepended with the assignment of the $i^{\text {th }}$ element of $\alpha^{2}$ to the given numeric variable and appended with an index increment statement, one final repetition of the loop body prepended with the assignment $i^{\text {th }}$ element of $\alpha^{2}$ but without the index increment, and finally the loop-unrolled continuation from the original loop. In terms of the above example, loop becomes

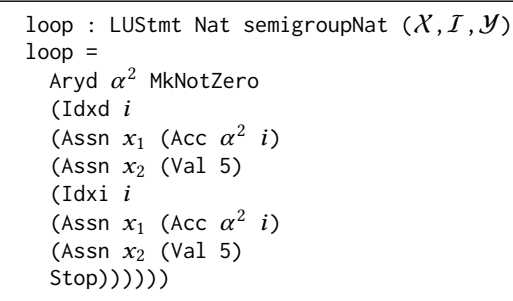

We note that the covering function for UnrollcPstmt returns a Maybe instead of a Dec due to difficulties of the type checker to reduce the fold in proofs of contradiction in the Iter case. Since we are only interested in the proofs for assertions in well-formed programs, providing a proof of why a given program is not well-formed is not strictly necessary and will be left to future work.

Index Reification. Following loop-unrolling, index variables are reified. We define a new statement representation, RStmt, that is the same as Lustmt barring three changes: index declaration and increment constructors no longer take arguments, and all other occurrences of index variables are replaced with their values taken from some index-context.

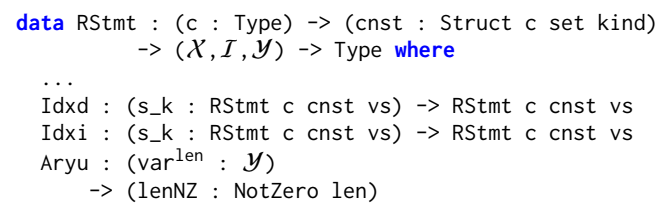

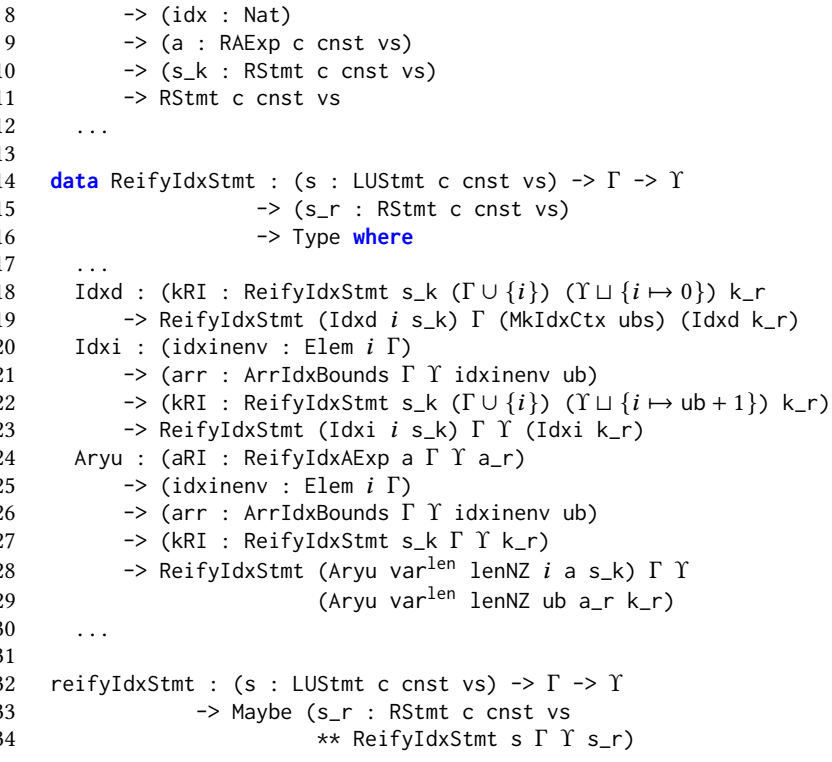

Here, ReifyIdxStmt relates a loop-unrolled statement with an equivalent index-reified statement. The environment, $\Gamma$, is only updated when an index variable is declared. Upon declaration of an index variable, the index context is also updated such that it maps the declared index variable, $i$, to 0 . Should $i$ already be in $\Gamma$, the new mapping replaces the old. In the above code, this is represented by ப; i.e. $\Upsilon \sqcup\left\{i_{j} \mapsto m\right\}=\Upsilon \backslash\left\{i_{j} \mapsto 0, i_{j} \mapsto 1, i_{j} \mapsto 2, \ldots\right\} \cup\left\{i_{j} \mapsto m\right\}$. Index increment statements update the context analogously. In our implementation, we do not remove old instances in either the environment or context, but instead rely upon the definition of isElem returning the first occurrence of $i$ in $\Gamma$. Future work will address this reliance upon implementation idiosyncrasies.

As in Section 3.4.5, the covering function, reifyIdxStmt, returns a Maybe value instead of a Dec due to impossible contradiction proof obligations. This is a consequence of the result that for any two proofs of vector membership, $p_{1}, p_{2}$ : Elem $\times \mathrm{xs}$, it does not hold that $p_{1}=p_{2}$, for a given $\times$ and $\times s$. Future work will address this.

Well-Formedness. The index-reification of statements enables the definition of a type expressing well-formedness; i.e. all variables are assigned/declared prior to occurrences in statements/subexpressions and that array accesses are never out-of-bounds.

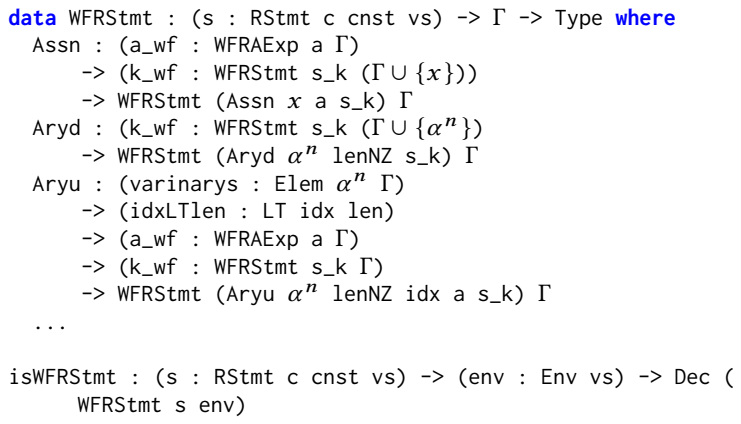


Here, the interesting cases are: numeric variable assignment and array declaration, which adds the relevant variable to the environment; and array update, which requires that the array being accessed is in the environment and that the index is strictly less than the length of the array (arrays are considered to be zero-indexed).

\subsection{Operational Semantics}

We define a big-step operational semantics for well-formed programs in IMP. Since (finite) well-formed programs are intended to be both guaranteed to be ground and terminate, no error states are produced during evaluation/execution.

3.5.1 Contexts. Similarly to index contexts in Section 3.4.2, we define a context, $\Psi$, for numeric and array variables.

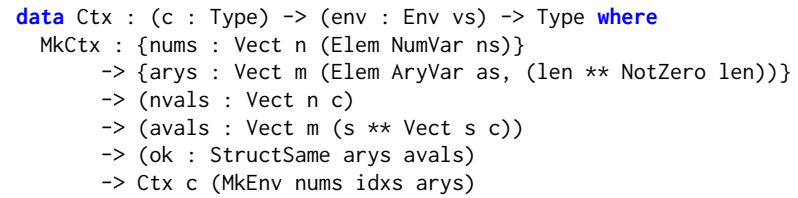

Here, a context takes a vector of numeric values, nvals, and a vector of array values, avals. Both vectors have the same length as the vector of numeric and array variables that are in the environment. The $i^{\text {th }}$ element in nvals and avals are mapped to the $i^{\text {th }}$ element in nums and arys, respectively. Each element in avals is itself a vector of numeric values. The proof term, (ok : StructSame arys avals), requires that each vector of numeric values in avals is the same length as the declared array in arys. Upon declaration, each element of an array is set to the zero value defined in the given setoid over c.

Example 3.11 (Context). We might define an example context for the environment defined in Example 3.7, which has a single numeric variable, $x_{2}$, and three arrays, $\alpha_{1}^{1}, \alpha_{2}^{3}$, and $\alpha_{2}^{5}$, in scope.

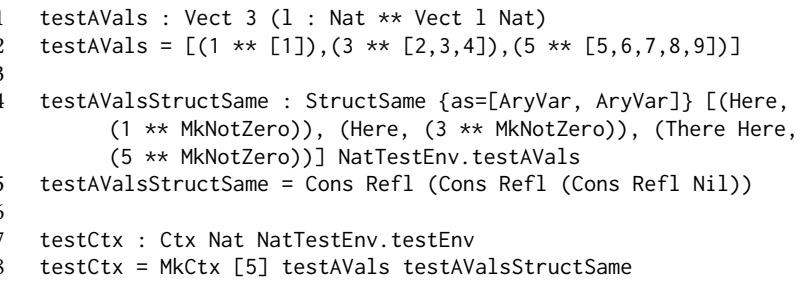

Here, $x_{2}=5 ; \alpha_{1}^{1}=[1] ; \alpha_{2}^{3}=[2,3,4]$; and $\alpha_{2}^{5}=[5,6,7,8,9]$. The variable testAValsStructSame provides the proof that the list of arrays, testAVals is a vector of length three, with nested vectors of lengths 1 , 3 , and 5 . We equivalently represent testctx as the set of substitutions

$$
\Psi=\left\{x_{2} \mapsto 5, \alpha_{1}^{1} \mapsto[1], \alpha_{2}^{3} \mapsto[2,3,4], \alpha_{2}^{5} \mapsto[5,6,7,8,9]\right\}
$$

3.5.2 Arithmetic Expressions. We define a big step operational semantics for algebraic expression via the type, SRAExp, which relates a given reified arithmetic expression to its value in c, given some context $\Psi$.

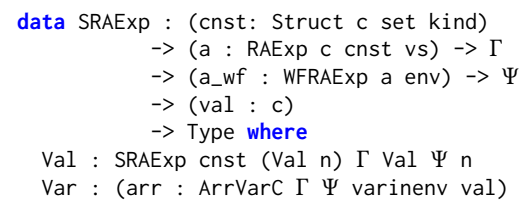

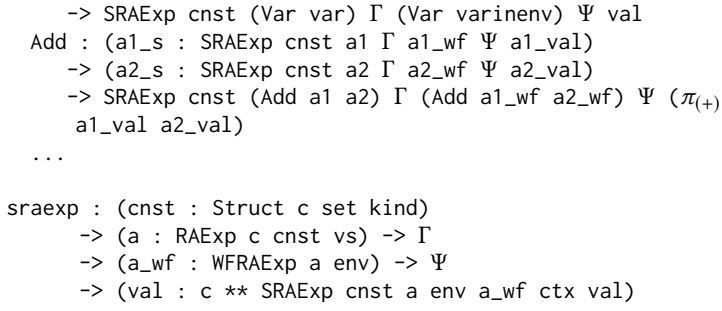

Literal values evaluate to themselves; variables and array accesses are related to a value in the context by ArrVarc and ArrVarIdxc, respectively; and addition, negation, and multiplication are evaluated using the respective functions defined in the given algebraic structure.

3.5.3 Boolean Expressions. The semantics for boolean expressions are defined analogously to arithmetic expressions above. $\mathrm{Nu}-$ meric sub-expressions are evaluated, and the functions provided for definitional equality and inequalities are projected from the given algebraic structure.

3.5.4 Statements. We define the semantics of statements via the type, SRStmt. Unlike expressions, the result of executing the statements is the context, $\Psi$. Specifically, the final state of a given statement is the context of the final continuation; i.e. the context provided to a stop statement.

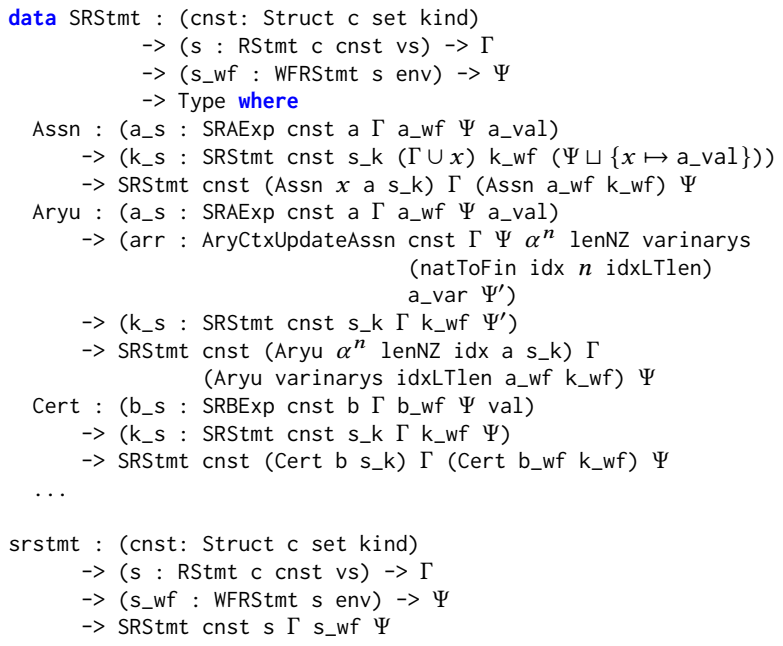

Here, the interesting cases are numeric variable assignment, array update, and $\mathrm{CSL}_{\mathrm{IMP}}$ assertions. Assignments require the evaluation of the arithmetic expression, a, the result of which is used to update the context. As in Section 3.5.1, $\sqcup$ denotes the addition or replacement of a mapping in a given context. In our implementation, as before, we simply prepend a_val to the list of numeric values in the context. Array updates are similar, requiring the evaluation of the arithmetic expression, which is then used to update the relevant value in the context. Here, the update is defined via the type, AryctxUpdateAssn. This relates the current context with a new context such that only the element at index idx of the array assigned to $\alpha^{n}$ is replaced by a_val. Array declarations are defined analogously, but such that a new vector of zero values are added 
to the context. Finally, we define the semantics of $\mathrm{CSL}_{\mathrm{IMP}}$ assertions to be equivalent to a typical skip statement. Assertions do not affect the execution of the program, but are instead used by the automatic proof inference system in order to determine whether the assertion holds. The argument, b_s, represents the evaluation of the boolean expression, and is used in order to determine the values of arithmetic sub-expressions when generating proofs for assertions.

\section{AUTOMATICALLY PROVING ASSERTIONS}

In this section, we define the type, vCRStmt, that extends assertion statements from CSL (see Section 2.1 and Listing 1 for an example of CSL assertions in C) with proofs (of contradition) that demonstrate that the assertion holds/does not hold true for the current context.

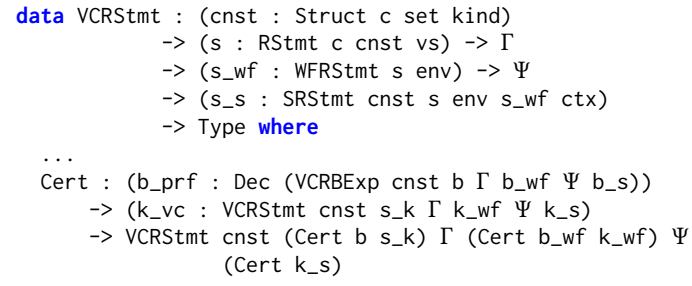

Naturally, cert is the only interesting case. It takes an argument that represents the proof of the (reified) Boolean expression that comprises the assertion for the environment and context at that particular statement. The environment and context are derived from, and defined by, the proofs of well-formedness and semantics that VCRStmt is indexed by. The proof itself is comprised of the type, VCRBexp, which represents only (propositionally) true Boolean expressions under the given context. Accordingly, and following convention, a proof that the assertion does not hold is represented by a function with the type VCRBExp cnst b $\Gamma$ b_wf $\Psi$ b_s $\rightarrow>$ Void.

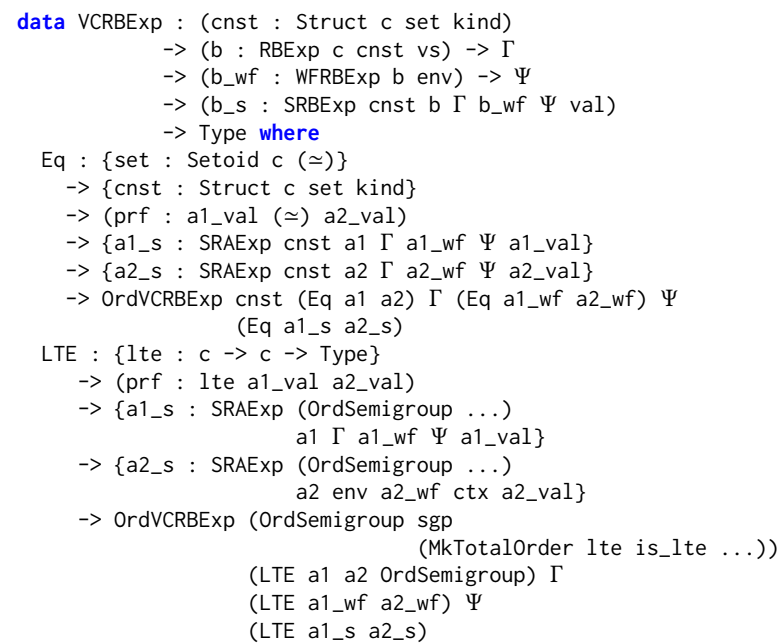

Here, both equality and inequality cases take the respective type definition from the given algebraic structure that represents the boolean operation. Each case then require an element of that type applied to the evaluated arithmetic sub-expressions. VCRBExp can be extended according to the defined algebraic structures and operations. The decision procedure for VCRBExp is straightforward.

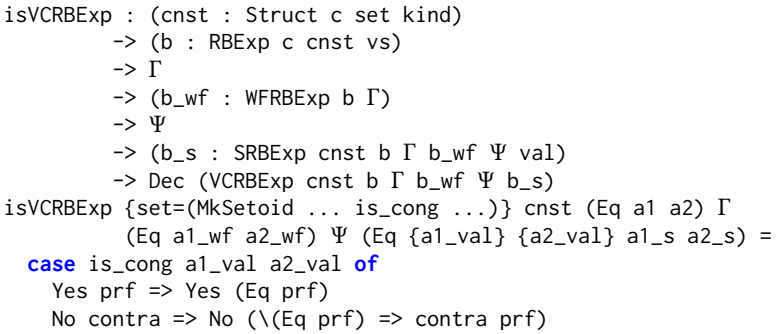

Here, the relevant decision procedure is projected from the given algebraic structure and is used to produce a proof, which is then used as an argument to the concomitant VCRStmt constructor.

\section{DEMONSTRATION}

In order to illustrate our approach, we consider list summation, an example of summing an array of natural numbers to demonstrate the principles of our technique, where we manually apply each step; it is intended that this process will be fully automatic in the future. In $\mathrm{C}$, we might have the summation function, sumList, that takes

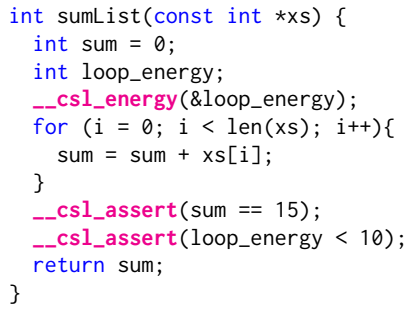

an array as a parameter and returns the sum of its elements. Here, sumList has been annotated with a CSL capture annotation on Line 4 to measure, e.g., the estimated energy consumption of the forstatement on Lines 5-7. CSL assertions have also been introduced on Lines $8 \& 9$; the first represents a check that the result of the loop is correct, and the second represents that the cost of the loop is within a given upper-bound, i.e. does not exceed 10 joules of energy. The body of sumList can be represented in IMP for a specific value of $x$ and where we assume, for the sake of this example, that all values in $x s$ are zero or greater (i.e. are natural numbers).

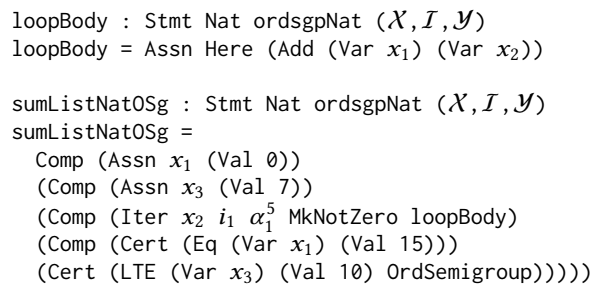

We use the ordsemigroup definition for the natural numbers from Example 3.3 and assume the existence of the array $[1,2,3,4,5]$, whose declaration and assignment statements are omitted, in the environment and context. Here, $x_{1}$ is the accumulator, $x_{2}$ is assigned to each element in the array over the course of all iterations of the loop, and $x_{3}$ represents the result of the CSL capture annotation in 
Line 4 of the sumList definition. The assertion on Line 9 represents a functional check that the result of the summation is the expected value, and the assertion on Line 10 represents a check to ensure that the result of the capture annotation is within a certain bound. In order to generate proofs of these assertions, we first ensure that sumListNatosg is well-formed. This process begins with a transformation into continuation-passing style by the relstmtCPS covering function.

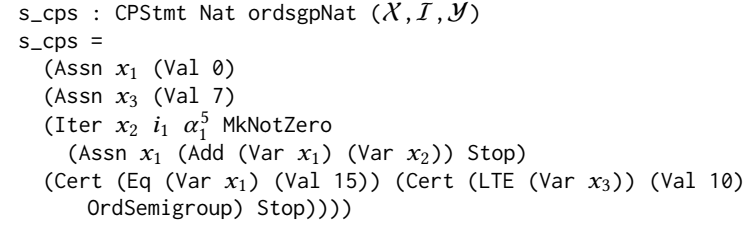

This is followed by loop-unrolling via the unrollcPstmt, which replicates the body of the loop five times.

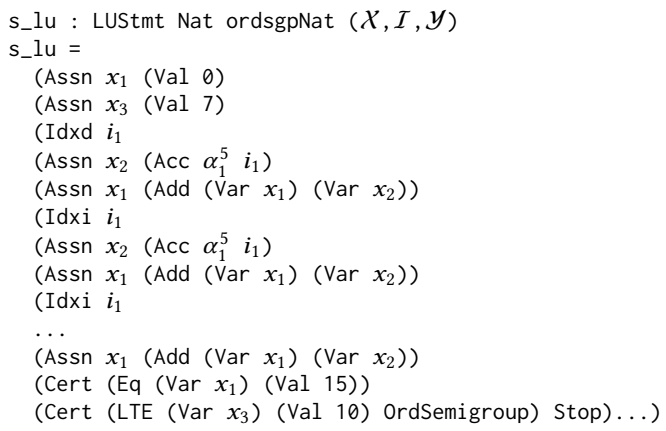

In order to maintain functional equivalence, the unrolled loop is preceded by a declaration of $i_{1}$ (Line 5), each repetition of the loop body is preceded by a statement assigning $x_{2}$ to the $i^{\text {th }}$ element of $\alpha_{1}^{5}$ (Lines $6 \& 9$ ), and finally, each repetition, excepting the last, of the loop body is followed by the incrementation of $i_{1}$ (Lines $8 \&$ 11). Having unrolled the loop, it is now possible to reify s_lu using reifyIdxStmt.

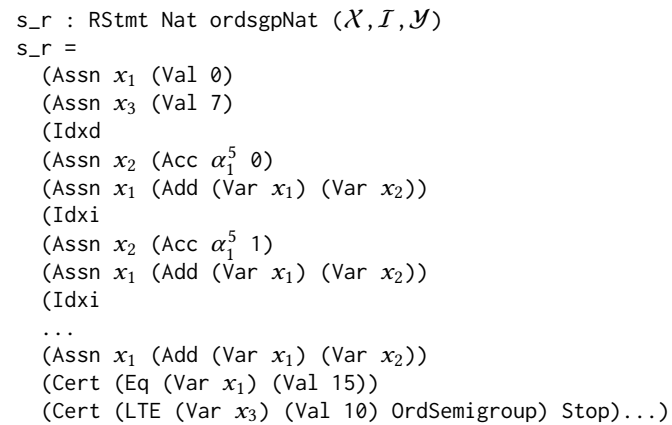

Here, we observe that the occurrences of $i_{1}$ in Lines $6 \& 9$ in s_lu have been replaced with their relevant natural numbers in Lines 6 $\& 9$ of s_r. We now determine the final stage of well-formedness by applying isWFRStmt.

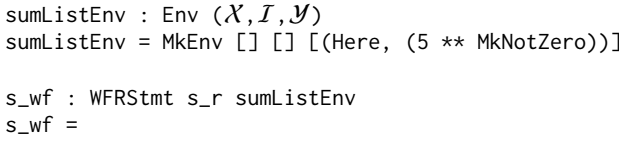

(Assn Val (Assn Val (Idxd

(Assn (Acc MkNotZero Here (LTESucc LTEZero))

(Assn (Add (Var (There (There Here))) (Var Here))

(Idxi

(Assn (Acc MkNotZero Here (LTESucc (LTESucc LTEZero)))

(Cert (Eq (Var Here) Val)

$($ Cert $(\operatorname{LTE}(\operatorname{Var}(\operatorname{There} \ldots($ There Here $))))))))))$ ) Val) Stop)...)

Since we assume that $\alpha_{1}^{5}$ is already defined and its elements assigned, we specify the initial environment sumListEnv, which only contains an array of length 5. s_wf is then a proof that all variables are in the environment before they occur in assignment statements or subexpressions (e.g. on Lines 7-8, 10, \& 12-13), and that array accesses are not out-of-bounds (e.g. on Lines $10 \& 13$ ). Given this proof that s_r is well-formed, and in order to determine whether the assertions hold, it is necessary to first derive a context for those assertions. We therefore apply srstmt to both s_r and s_wf. We note that this is equivalent to executing the program and using the values of $x_{1}$ and $x_{3}$ at the point of each assertion.

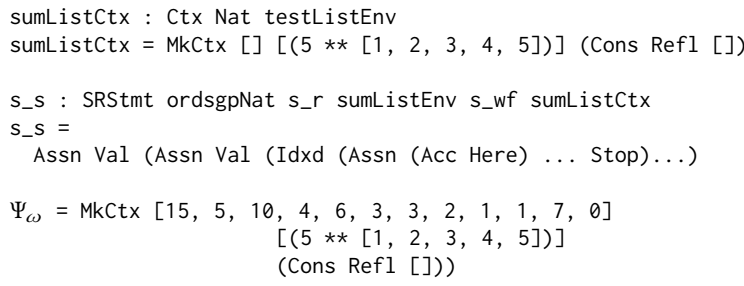

As before, we provide an initial context with our array already included. The result of srstmt is a witness to our semantics, s_s, and the inferred contexts at each continuation, where $\Psi_{\omega}$ is the final context. In $\Psi_{\omega}$, we observe that the values for the arrays have not changed, which is expected. Excepting the final two elements of the vector in Line 8, which represents the values of numeric variables, each pair of elements (i.e. $15 \& 5,10 \& 4$, etc.) represent updated values of $x_{1}$ and $x_{2}$ respectively. We can now generate the proofs for both assertion statements by applying vcrstmt to s_r, s_wf, and

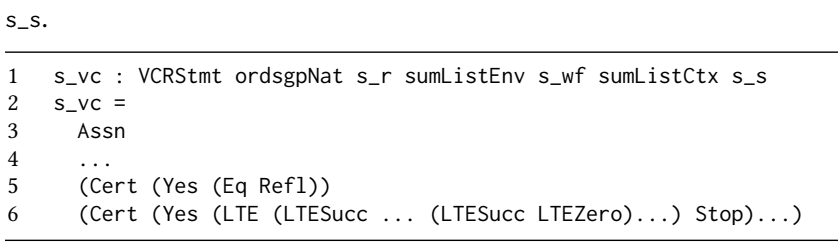

Here, s_vc represents the extension of assertions in s_r given the context at that point the program. Line 5 contains the proof that $x_{1} \simeq 15$ holds true, where $x_{1}=1+2+3+4+5$. Similarly, Line 6 contains the proof that the cost value obtained from the capture annotation is less than the programmer-provided upper bound; i.e. $x_{3} \leq 10$, where $x_{3}=7$.

\section{RELATED WORK}

In addition to the aforementioned related work throughout the paper, the calculation and bounding of resource usage is a topic of great interest in the programming language community, with approaches typically focussed on time, space, and type systems [8, $9,15,17,18,21,32]$. Other non-functional properties, such as information flow and leakage, have also been modelled using type 
systems [7, 33]. Energy consumption, which is of increasing interest to embedded systems programming [24], has also been represented as a function of program arguments [13, 22]. These approaches typically depend upon specific type systems or languages; accordingly, applying them to other languages can prove non-trivial [6]. Abstract interpretation offers an alternative approach to the verification and debugging of programs in languages that may not necessarily be best equipped for the desired techniques [23]. Examples include debugging of both imperative and logical programs [3, 4], and approaches to verification by Cousot [10]. More recently, The Ciao Preprocessor system (CiaoPP) [16, 23, 29] models Java [14] and $\mathrm{XC}$ [1] programs as sequences of Horn Clauses in order to debug and certify programs, using resource usage information that the system derives. A high-level comparison between CiaoPP and Drive is given by Brown et al. [6].

\section{CONCLUSIONS AND FUTURE WORK}

In this paper, we presented the small generic imperative language, IMP, representing a subset of C with CSL assertions. Using the dependently-typed language, Idris, we defined both the syntax and a big-step operational semantics for IMP. IMP is parameterised by a pointed carrier type and an algebraic structure, enabling a generic and formally-based framework for the expression of arithmetic and Boolean operations. Our semantics for IMP facilitate a robust context inference that, in turn, facilitates the automatic generation of proofs for CSL assertions. We demonstrate our approach on a representative example of summing an array, featuring both CSL assertions and capture annotations.

In the future, we will expand our evaluation to include the entirety of the BEEBs benchmark suite, demonstrating a range of nonfunctional properties, including both energy and time. Furthermore, we will extend our non-functional properties to include security, allowing our formalism to guide the programmer in preventing common security hacks, such as side-channel attacks. Finally, we will prove properties of both our semantics and abstract interpretation, including soundness, determinism, and confluence, in order to improve confidence in our approach.

\section{ACKNOWLEDGEMENTS}

This work was generously supported by the EU Horizon 2020 project, TeamPlay (https://www.teamplay-h2020.eu), grant number 779882, and UK EPSRC Discovery, grant number EP/P020631/1.

\section{REFERENCES}

[1] 2011. XC Specification ver. 1.0 (X5965A) https://www.xmos.com/developer/xc-specification.

[2] Elvira Albert, Puri Arenas, Germán Puebla, and Manuel V. Hermenegildo. 2012 Certificate size reduction in abstraction-carrying code. TPLP 12, 3 (2012), 283318.

[3] Giovanni Bacci and Marco Comini. 2010. Abstract Diagnosis of First Order Functional Logic Programs. In LOPSTR (Lecture Notes in Computer Science), Vol. 6564. Springer, 215-233.

[4] François Bourdoncle. 1993. Abstract Debugging of Higher-Order Imperative Languages. In PLDI. ACM, 46-55.

[5] Edwin Brady. 2013. Idris, a general-purpose dependently typed programming language: Design and implementation. F. Funct. Program. 23, 5 (2013), 552-593.

[6] Christopher Brown, Adam D. Barwell, Yoann Marquer, Céline Minh, and Olivier Zendra. 2019. Type-Driven Verification of Non-functional Properties. In PPDP. Accepted for publication.
[7] Hongxu Chen, Alwen Tiu, Zhiwu Xu, and Yang Liu. 2018. A PermissionDependent Type System for Secure Information Flow Analysis. In CSF. IEEE Computer Society, 218-232.

[8] Wei-Ngan Chin and Siau-Cheng Khoo. 2001. Calculating Sized Types. HigherOrder and Symbolic Computation 14, 2-3 (2001), 261-300.

[9] Ezgi Çiçek, Deepak Garg, and Umut A. Acar. 2015. Refinement Types for Incremental Computational Complexity. In ESOP (Lecture Notes in Computer Science), Vol. 9032. Springer, 406-431.

[10] Patrick Cousot. 2003. Automatic Verification by Abstract Interpretation. In VMCAI (Lecture Notes in Computer Science), Vol. 2575. Springer, 20-24.

[11] Patrick Cousot and Radhia Cousot. 1977. Abstract Interpretation: A Unified Lattice Model for Static Analysis of Programs by Construction or Approximation of Fixpoints. In POPL. ACM, 238-252.

[12] Heiko Falk and Paul Lokuciejewski. 2010. A compiler framework for the reduction of worst-case execution times. Real-Time Systems 46, 2 (01 Oct 2010), 251-300. https://doi.org/10.1007/s11241-010-9101-x

[13] Kyriakos Georgiou, Steve Kerrison, Zbigniew Chamski, and Kerstin Eder. 2017. Energy Transparency for Deeply Embedded Programs. TACO 14, 1 (2017), 8:18:26.

[14] James Gosling, Bill Joy, Guy L. Steele, Gilad Bracha, and Alex Buckley. 2014. The fava Language Specification, Java SE 8 Edition (1st ed.). Addison-Wesley Professional.

[15] Sumit Gulwani, Krishna K. Mehra, and Trishul M. Chilimbi. 2009. SPEED: precise and efficient static estimation of program computational complexity. In POPL. ACM, 127-139.

[16] Manuel V. Hermenegildo, Germán Puebla, Francisco Bueno, and Pedro LópezGarcía. 2005. Integrated program debugging, verification, and optimization using abstract interpretation (and the Ciao system preprocessor). Sci. Comput. Program. 58, 1-2 (2005), 115-140.

[17] Jan Hoffmann, Klaus Aehlig, and Martin Hofmann. 2012. Multivariate amortized resource analysis. ACM Trans. Program. Lang. Syst. 34, 3 (2012), 14:1-14:62.

[18] John Hughes and Lars Pareto. 1999. Recursion and Dynamic Data-structures in Bounded Space: Towards Embedded ML Programming. In ICFP. ACM, 70-81.

[19] Graham Hutton. 2007. Programming in Haskell. Cambridge University Press, New York, NY, USA.

[20] Paul C. Kocher. 1996. Timing Attacks on Implementations of Diffie-Hellman, RSA, DSS, and Other Systems. In CRYPTO (Lecture Notes in Computer Science), Vol. 1109. Springer, 104-113.

[21] Ugo Dal Lago and Barbara Petit. 2013. The geometry of types. In POPL. ACM, 167-178.

[22] Umer Liqat, Steve Kerrison, Alejandro Serrano, Kyriakos Georgiou, Pedro LópezGarcía, Neville Grech, Manuel V. Hermenegildo, and Kerstin Eder. 2013. Energy Consumption Analysis of Programs Based on XMOS ISA-Level Models. In LOPSTR (Lecture Notes in Computer Science), Vol. 8901. Springer, 72-90.

[23] Pedro López-García, Luthfi Darmawan, Maximiliano Klemen, Umer Liqat, Francisco Bueno, and Manuel V. Hermenegildo. 2018. Interval-based resource usage verification by translation into Horn clauses and an application to energy consumption. TPLP 18, 2 (2018), 167-223.

[24] Jeremy Morse, Steven Kerrison, and Kerstin Eder. 2018. On the limitations of analysing worst-case dynamic energy of processing. ACM Transactions on Embedded Computing Systems 17, 3 (2 2018). https://doi.org/10.1145/3173042

[25] George C. Necula. 1997. Proof-Carrying Code. In POPL. ACM Press, 106-119.

[26] Hanne Riis Nielson and Flemming Nielson. 2007. Semantics with Applications: An Appetizer. Springer.

[27] James Pallister, Simon J. Hollis, and Jeremy Bennett. 2013. BEEBS: Open Benchmarks for Energy Measurements on Embedded Platforms. CoRR abs/1308.5174 (2013). arXiv:1308.5174 http://arxiv.org/abs/1308.5174

[28] Christopher Schwaab, Ekaterina Komendantskaya, Alasdair Hill, Frantisek Farka, Ronald P. A. Petrick, Joe B. Wells, and Kevin Hammond. 2019. Proof-Carrying Plans. In PADL (Lecture Notes in Computer Science), Vol. 11372. Springer, 204-220.

[29] Alejandro Serrano, Pedro López-García, and Manuel V. Hermenegildo. 2014. Resource Usage Analysis of Logic Programs via Abstract Interpretation Using Sized Types. TPLP 14, 4-5 (2014), 739-754.

[30] Franck Slama and Edwin Brady. 2017. Automatically Proving Equivalence by Type-Safe Reflection. In CICM (Lecture Notes in Computer Science), Vol. 10383. Springer, 40-55.

[31] The Univalent Foundations Program. 2013. Homotopy Type Theory: Univalent Foundations of Mathematics. https://homotopytypetheory.org/book, Institute for Advanced Study.

[32] Pedro B. Vasconcelos and Kevin Hammond. 2003. Inferring Cost Equations for Recursive, Polymorphic and Higher-Order Functional Programs. In IFL (Lecture Notes in Computer Science), Vol. 3145. Springer, 86-101.

[33] Dennis M. Volpano, Cynthia E. Irvine, and Geoffrey Smith. 1996. A Sound Type System for Secure Flow Analysis. Journal of Computer Security 4, 2/3 (1996), 167-188. 